\title{
Immobilization of Cellulase in the Swollen Microsphere by Radiation Polymerization
}

\author{
Masaru Yoshida, ${ }^{*}$ Minoru Kumakura, ${ }^{*}$ and Isao Kaetsu* \\ *Takasaki Radiation Chemistry Research Establishment, \\ Japan Atomic Energy Research Institute, \\ Takasaki, Gunma 370-12, Japan.
}

(Received March 12, 1979)

\begin{abstract}
The immobilization of cellulase in hydrophobic particles was carried out by the radiation polymerization of glycidyl methacrylate as a glass-forming monomer in the presence of poly(styrene) at low temperatures. When the monomer system containing cellulase was poured into cold alcohol, spheres were formed. Then, the rigid microspheres polymerized by irradiation were swollen with acetone, benzene or toluene into soft elastic matrices. The activity yield of immobilized cellulase in swollen matrix increased to about $4-6$ times than that of the rigid particles. The microscopic observation proved that the swelling treatment formed many wrinkles on the expanded particle surface, thus increasing the surface area greatly.

KEY WORDS Immobilization / Cellulase / Radiation Polymerization / Swollen Matrix / Microsphere / Glycidyl Methacrylate / Polystyrene /
\end{abstract}

The authors have studied radiation polymerization at low temperatues using glass-forming monomers. ${ }^{1}$ Biofunctional polymer materials including enzymes, ${ }^{2}$ microbial cells, ${ }^{3}$ organelles, ${ }^{4}$ and drugs $^{5-6}$ were developed as applications of this polymerization. Glass-forming monomers are advantageous for low-temperature polymerization due to their stable supercooling property and high polymerizability.

Various biofunctional substances and livings can be entrapped effectively on vinyl polymer of various forms by mixing with monomer shaping and irradiating by radiation at low temperatures. This method is utilizable for purposes of immobilization and controlled slow release of bio-components. Entrapping of bio-components on polymer surface, especially on a surface of hydrophobic polymer particle, is one of the characteristic feature of this method. Therefore, the obtained material can be used advantageously for the surface reaction with the substrate of high molecular weight or waterinsoluble property. The preparation of a hydrophobic polymer microsphere containing enzymes or microbial cells has already been studied by radiation suspension polymerization of glass-forming monomers. ${ }^{8}$ However, effective surface area of polymer sphere for enzyme reaction is a very important factor for the activity of the material.

In this report, an improved immobilization method in hydrophobic microsphere form containing cellulase with increased surface area has been proposed which comprises radiation polymerization of glycidyl metacrylate as a glass-forming monomer in the presence of vinyl polymer at low temperatures.

\section{EXPERIMENTAL}

\section{Materials}

Enzyme. Cellulase from Trichoderma viride was obtained from Kinki Yakult MFG Co., Ltd.

Glass-Forming Monomers. Glycidyl methacrylate (GMA), product of Tokyo Kasei Kogyo Co., Ltd. was purified by distillation according to conventional methods. Diethylene glycol dimethacrylate (DGDA) and trimethylolpropane trimethacrylate (TMPT) were obtained from Shin-Nakamura Chemical Co., Ltd. and purified by the same method in a previous report. ${ }^{5}$

Polymer. Polystyrene (PST) was obtained from Kishida Chemical Co., Ltd. Its degree of polymerization is about 1700 .

Precipitation Media. Methanol, ethanol, pro- 
panol, acetone, toluene, and tetrahydrofuran were obtained from Tokyo Kasei Kogyo Co., Ltd. and used without further purification.

Swelling Solvents. Acetone, benzene, and toluene from Tokyo Kasei Kogyo Co., Ltd. were used without purification.

Substrate. Carboxymethyl cellulose (sodium salt) (CMC), $n=500$, was obtained from Kanto Chemical Co., Ltd. A $0.5 \%$ CMC solution $(0.1 \mathrm{M}$ acetate buffer solution, $\mathrm{pH} 4.5$ ) was used for enzyme reaction.

\section{Preparation of Radiation Polymerized Particles}

PST was completely dissolved in GMA at 40 $80^{\circ} \mathrm{C}$. One $\mathrm{ml}$ of the PST-GMA solution was mixed with $5 \mathrm{mg}$ of cellulase. The obtained enzymemonomer suspension was dropped into a cold precipitation medium with a pipette ( $1 \mathrm{~mm}$ fine tip). The supercooled glassy particles in the precipitation media was irradiated at $-78^{\circ} \mathrm{C}$ for 2 hours at a dose rate of $5 \times 10^{5} \mathrm{R} \mathrm{h}^{-1}$, with $\gamma$-ray from a ${ }^{60} \mathrm{Co}$ source. The dried polymer particles were then treated with a swelling solvent at room temperature.

The mean diameter of a dried particle $\left(D_{\text {av }}\right)$ was measured by an optical microscope, Model SMZ-6, Nippon Kogaku Co., Ltd. The cellulase content per particle $\left(W_{\mathrm{e}}\right)$ was estimated from the initial enzyme concentration and the number of particles $\left(N_{\mathrm{p}}\right)$ which was read by microscopic observation. The degree of swelling $\left(S_{\mathrm{w}}\right)$ was determined according to the following equation;

$$
S_{\mathrm{w}}=\frac{W_{\mathrm{s}}}{W_{\mathrm{p}}}
$$

where $W_{\mathrm{s}}$ and $W_{\mathrm{p}}$ are the weight of solvent absorbed in the polymer particle and that of the particle.

\section{Assay of Cellulase Activity}

The swollen particles were washed for 48 hours at $25-35^{\circ} \mathrm{C}$ with $1000 \mathrm{ml}$ of ethanol and then with $1000 \mathrm{ml}$ of $0.1 \mathrm{M}$ acetate buffer solution ( $\mathrm{pH} 4.5$ ) to remove the solvent. The immobilized enzyme was shaken with $10 \mathrm{ml}$ of $0.5 \% \mathrm{CMC}$ solution ( $\mathrm{pH} \mathrm{4.5)} \mathrm{at}$ $40^{\circ} \mathrm{C}$ for 60 minutes. The glucose formed was measured by absorption at $505 \mathrm{~nm}$ with a Shimazu double beam spectrophotometer, Model UV-200, and by the glucose oxidase-peroxidase-chlomogen method. ${ }^{2}$ The enzyme reaction was carried out repeatedly.

The activity yield of the immobilized cellulase may be represented as follows.

$$
\text { Activity yield }(\%)=\frac{I_{\mathrm{a}}}{N_{\mathrm{a}}} \times 100
$$

where $I_{\mathrm{a}}$ is the quantity of hydrolyzed product by the cellulase during each batch reaction and $N_{\mathrm{a}}$ is the quantity of the product formed by native cellulase.

\section{Measurement of Particle Structure}

The particle structure was observed with a scanning electron microscope, Model JSM-03, Japan Electron Optics Laboratory Co., Ltd.

\section{RESULTS AND DISCUSSION}

\section{Trapping of Cellulase by Polymerization of GMA in the Presence of PST}

The mixture of PST, GMA, and cellulase was added to various medium below $-24^{\circ} \mathrm{C}$ to make particle through a nozzle and the resuls are summarized in Table I. The use of a glass-forming monomer such as GMA is advantageous, since the coagulation of monomeric micro-particles can be prevented owing to the high viscosity of supercooled solution. The addition of polymer such as PST insoluble to the precipitation media is necessary to cover the monomer particle with polymer skin to keep it from being dissolved in the medium. Almost perfect spherical particles were obtained when alcohols such as methanol, ethanol, and propanol were used as the shaping media, though no particle formed without the addition of PST. However, acetone, toluene and tetrahydrofuran were not suitable as shaping media even at $-78^{\circ} \mathrm{C}$, no particles. could form owing to the considerable solubility of PST skin in these media. The optical photograph of polymerized spherical particles (sample 2 in Table I) is shown in Figure 3(a). However, it is important to further increase the surface area of these particles for more efficient contact of the substrate with enzyme. For this purpose, the polymerized particles was swollen by acetone, toluene and benzene. The swollen particles changed from a rigid matrix to an expanded elastic marshmallow-like matrix in shaping and softness.

The absorbed swelling agents were gradually washed away with water from polymer matrix while using the particles for an enzyme reaction with an aqueous suspension of substrate. As the result, the particles having large surface areas due to wrinkles 
Immobilization of Cellulase in the Swollen Microsphere

Table I. Preparation of spherical particles in radiation polymerization at low temperatures

\begin{tabular}{|c|c|c|c|c|c|c|}
\hline \multirow{2}{*}{ Sample } & \multirow{2}{*}{ Monomer system } & \multicolumn{2}{|c|}{ Preparation condition } & \multicolumn{3}{|c|}{ Property of polymerized particle } \\
\hline & & Medium & Temperature $/{ }^{\circ} \mathrm{C}$ & $D_{\mathrm{av}} / \mathrm{mm}$ & $N_{\mathrm{p}} /$ particles & $W_{\mathrm{e}} / \mu \mathrm{g} /$ particle $^{-1}$ \\
\hline 1 & & Methanol & -78 & 3.2 & 38 & 132 \\
\hline 2 & & Ethanol & -78 & 3.0 & 42 & 119 \\
\hline 3 & & Propanol & -78 & 3.4 & 35 & 143 \\
\hline 4 & $10 \%$ PST $-90 \%$ GMA & Acetone & -78 & \multicolumn{3}{|c|}{ Nonparticle (thin membrane-like composite) } \\
\hline 5 & & Toluene & -78 & \multicolumn{3}{|c|}{ Nonparticle (curdy composite) } \\
\hline 6 & & Tetrahydrofuran & -78 & \multicolumn{3}{|c|}{ Nonparticle (ring-like composite) } \\
\hline 7 & & Methanol & 0 & \multicolumn{3}{|c|}{ Nonparticle (block composite) } \\
\hline 8 & $10 \%$ PST $-90 \%$ DGDA & Ethanol & -78 & 3.1 & 38 & 132 \\
\hline 9 & $10 \%$ PST $-90 \%$ TMPT & Ethanol & -78 & 3.0 & 44 & 114 \\
\hline
\end{tabular}

and micropores were formed. However, in the case of polyfunctional monomers such as DGDA and TMPT, the polymer particles were hardly swollen by any swelling agent owing to highly cross-linked structure of the polymers.

\section{Activity Yield of Cellulase in the Hydrophobic Particles}

The activity yield of cellulase in the hydrophobic particles are plotted against the number of batch enzyme reaction (repeated use) in Figure 1.

It is remarkable that the activity yield did not change with repeated use as in a previous work. ${ }^{7}$ However, the activity yield in swollen particles was about 4-6 times larger than that of rigid particles before the swelling treatment. The activity yield increases with increase in the degree of swelling for all cases in Figure 2. Acetone was the most effective solvent having the greatest capacity of all the solvents to induce swelling. The saturated $S_{\mathrm{w}}$ values in acetone, benzene, and toluene were 5.0, 3.6, and 2.7, respectively. This increase in activity through swelling may be attributed to the increase in the surface area owing to the wrinkles and porous structures in the matrix. That is, certain active sites of enzyme occuluded in the matrix are perhaps exposed to enhance activity by surface area increase. At the same time, the substrate might diffuse into the inner surface of the matrix through the wrinkles and porous structures more easily and frequently to make contact with the enzyme in consequence of this increase. Since the substrate can hardly diffuse inside hydrophobic matrix, the enzymatic reaction has to

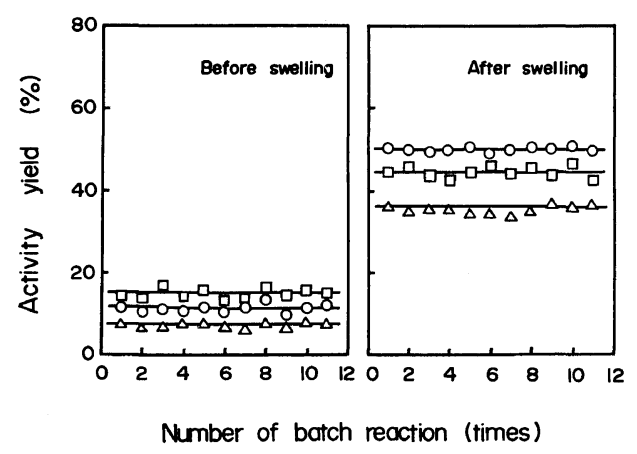

Figure 1. Relationship between the activity yield of cellulase in swollen particles and the repeated use. The polymerized particles (see, samples $1-3$ in Table I) were treated at room temperature with various swellingsolvents. The $S_{w}$ values were prepared to be about 2.5 in all cases. Swelling-solvent: $\bigcirc$, acetone (sample 1); $\square$, toluene (sample 2); $\triangle$, benzene (sample 3).

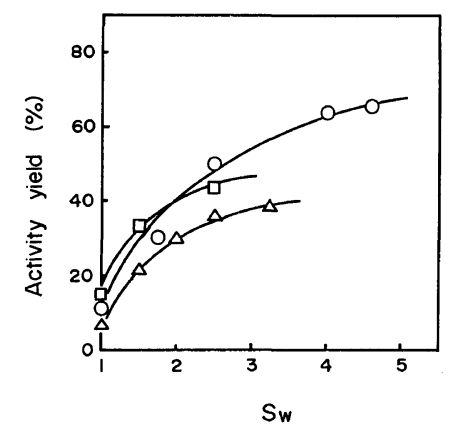

Figure 2. Relationship between the activity yield of cellulase in swollen particles and the degree of swelling $\left(S_{w}\right)$. Swelling-solvent: $\bigcirc$, acetone; $\square$, toluene; $\triangle$, benzene. 
be carried out on the matrix surface. Moreover, the swollen particles can be used repeatedly with no activity decrease due to enzyme leakage. Consequently, the swelling technique is the most advantageous method for increasing the activity of hydrophobic immobilized particles without lessening the other benefits of the hydrophobic carrier as well as the stable activity retention in repeated use. However, the activity yield for particles of $10 \%$ PST $-90 \%$ DGDA and $10 \%$ PST- $90 \%$ TMPT systems did not increase after treatment by solvent. The activity yield was only $5-10 \%$ of the swollen particles.

\section{Observation of Particle Structure}

The. structure of a hydrophobic particle was observed by microscopic photograph. After swelling $\left(S_{\mathrm{w}}=2.5\right)$, the particle size became about 2 times that of the original particle (before swelling) as shown in Figures 3 (optical microphotograph) and 4 (electron microphotograph).

The surface of expanded elastic particles swollen with acetone is apparently contracted by contact with water to form many wrinkles and micropore structures of $1-3 \mu \mathrm{m}$ in diameter. It is obvious that the observed wrinkles and micropores as well as volume expansion greatly increases the surface area of the matrix. These results support the explanation described above owing to the activity increase in the present method.

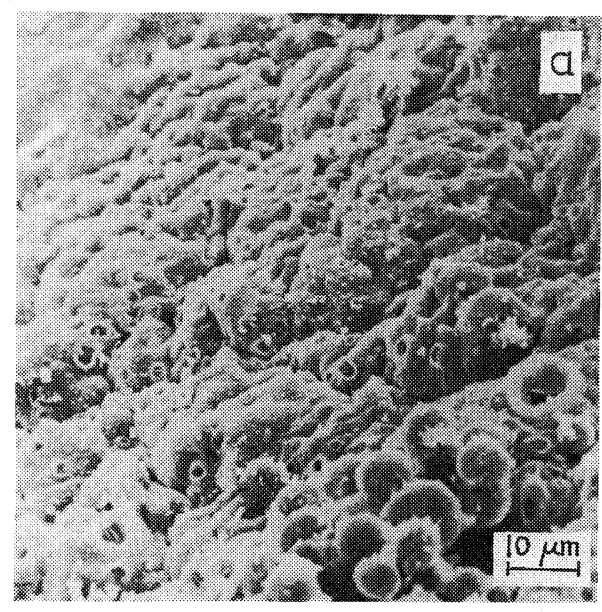

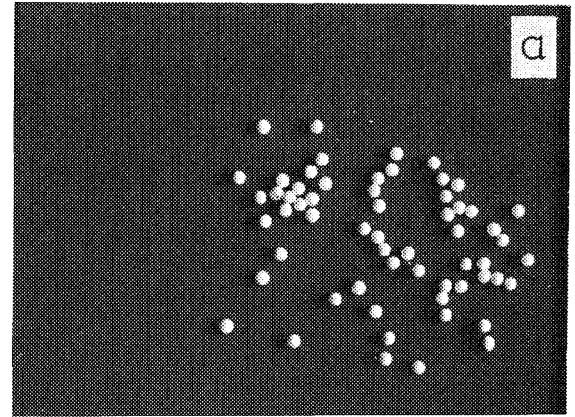

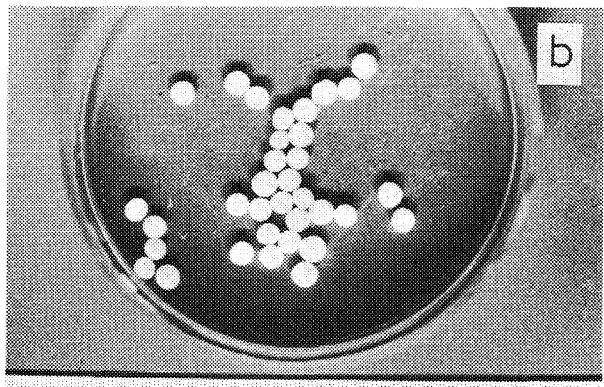

Figure 3. Optical microphotographs of swollen particles. The rigid particles were treated at room temperature for 16 hours with acetone as the swelling-solvent $\left(S_{w}=2.5\right)$ : (a) rigid particles before swelling; (b) expanded elastic particles after swelling (acetone only).

Figure 4. Scanning electron microphotographs of swollen particles. After swelling (see, (b) in Figure 3), the expanded elastic particles were washed with excess ethanol and then with excess water. After drying, the particle structure was observed by a scanning electron microscope. (a) $\times 1,500$; (b) $\times 15,000$. 
Acknowledgement. The authors are grateful to Dr T. Seguchi of our establishment for his assistance in making measurements by the electron microscope.

\section{REFERENCES}

1. I. Kaetsu, H. Okubo, A. Ito, and K. Hayashi, $J$. Polym. Sci., A-1, 10, 2203 (1972).

2. M. Yoshida, M. Kumakura, and I. Kaetsu, Polymer, 20, 3 (1979).

3. M. Kumakura, M. Yoshida, and I. Kaetsu, J. Solid-
Phase Biochem., 2, 279 (1978).

4. T. Fujimura and I. Kaetsu, Plant Physiol., in press.

5. M. Yoshida, M. Kumakura, and I. Kaetsu, Polymer, 19, 1375 (1978).

6. M. Yoshida, M. Kumakura, and I. Kaetsu, J. Pharm. Sci., 68, 628 (1979).

7. M. Kumakura, M. Yoshida, and I. Kaetsu, Eur. J. Appl. Microbiol. Biotechnol., 6, 13 (1978).

8. M. Yoshida, M. Kumakura, and I. Kaetsu, $J$. Macromol. Sci. Chem., in press. 